\title{
Typhoid fever and paratyphoid fever:
} Systematic review to estimate global morbidity and mortality for 2010

\author{
Geoffrey C. Buckle, \\ Christa L. Fischer Walker, \\ Robert E. Black
}

Johns Hopkins University Bloomberg School of Public Health, Baltimore, Maryland, USA

\section{Correspondence to:}

Christa L. Fischer Walker Johns Hopkins Bloomberg School of Public Health

615 North Wolfe Street, Rm E5608

Baltimore, MD 21205, USA

cfischer@jhsph.edu
Background Typhoid and paratyphoid fever remain important causes of morbidity worldwide. Accurate disease burden estimates are needed to guide policy decisions and prevention and control strategies.

Methods We conducted a systematic literature review of the PubMed and Scopus databases using pre-defined criteria to identify population-based studies with typhoid fever incidence data published between 1980 and 2009. We also abstracted data from annual reports of notifiable diseases in countries with advanced surveillance systems. Typhoid and paratyphoid fever input data were grouped into regions and regional incidence and mortality rates were estimated. Incidence data were extrapolated across regions for those lacking data. Age-specific incidence rates were derived for regions where age-specific data were available. Crude and adjusted estimates of the global typhoid fever burden were calculated.

Results Twenty-five studies were identified, all of which contained incidence data on typhoid fever and 12 on paratyphoid fever. Five advanced surveillance systems contributed data on typhoid fever; 2 on paratyphoid fever. Regional typhoid fever incidence rates ranged from $<0.1 / 100000$ in Central and Eastern Europe and Central Asia to $724.6 / 100000$ in Sub-Saharan Africa. Regional paratyphoid incidence rates ranged from 0.8/100 000 in North Africa/Middle East to 77.4/100 000 in Sub-Saharan Africa and South Asia. The estimated total number of typhoid fever episodes in 2010 was 13.5 million (interquartile range 9.1-17.8 million). The adjusted estimate accounting for the low sensitivity of blood cultures for isolation of the bacteria was 26.9 million (interquartile range 18.3-35.7 million) episodes. These findings are comparable to the most recent analysis of global typhoid fever morbidity, which reported crude and adjusted estimates of 10.8 million and 21.7 million typhoid fever episodes globally in 2000 .

Conclusion Typhoid fever remains a significant health burden, especially in low- and middle-income countries. Despite the availability of more recent data on both enteric fevers, additional research is needed in many regions, particularly Africa, Latin America and other developing countries. 
Typhoid and paratyphoid fever remain important public health problems globally and major causes of morbidity in the developing world [1]. Typhoid and paratyphoid fever are acute and often life-threatening febrile illnesses caused by systemic infection with the bacterium Salmonella enterica serotype typhi and paratyphi, respectively. Classical symptoms include gradual onset of sustained fever, chills, hepatosplenomegaly and abdominal pain. In some cases, patients experience rash, nausea, anorexia, diarrhea or constipation, headache, relative bradycardia and reduced level of consciousness [2]. While both diseases share clinical features, paratyphoid fever tends to have a more benign course of illness. Without effective treatment, typhoid fever has a case-fatality rate of $10-30 \%$. This number is reduced to $1-4 \%$ in those receiving appropriate therapy [1]

The most recent global burden of disease estimates for typhoid and paratyphoid fever reported that in 2000 , there were 22 million new cases of typhoid fever, 210000 typhoid fever-related deaths, and 5.4 million cases of paratyphoid fever [1]. This study offered improved estimates from past updates and analyses [1,3-6].

A revised estimate of the global burden of typhoid and paratyphoid fever is critically needed for developing improved strategies for disease prevention and control. The global epidemiology of these diseases has changed with global population growth and provision of clean water and sanitation systems. Advances in surveillance, improved understanding of the age distribution of the disease, and more recent studies allow for updated estimates of the global burden of typhoid and paratyphoid fever.

\section{METHODS}

\section{Systematic review and data extraction}

We conducted literature searches in PubMed and Scopus databases using combinations of the following search terms: typhoid; Salmonella typhi; Salmonella paratyphi; incidence; prevalence; mortality; disease burden; surveillance; distribution. The initial literature search was conducted in January 2009 and was updated on December 31, 2009. We screened study titles and abstracts focused on typhoid and/ or enteric fever according to a priori inclusion and exclusion criteria. For papers not excluded based on title and abstract, full text articles were obtained and reevaluated for inclusion/exclusion criteria. We sought to include all studies published from 1980-2009 collecting prospective, population-based typhoid fever incidence data with blood culture confirmation of diagnosis from both active and passive surveillance studies. Intervention studies were included, but estimates were based on non-intervention groups only. Studies published in English, Spanish, Italian, Portuguese, or French were included. We excluded studies that did not diagnose typhoid fever by blood culture or that used stool culture for diagnosis. We also excluded case reports, microbiological reports, studies of carriers, and studies whose results did not allow for separation of S. typhi and S. paratyphi cases. Studies of hospitalized patients were excluded unless differentiation between inpatients and outpatients was clear; however, studies that screened for typhoid fever among individuals presenting with febrile illness at clinics/ hospitals were considered separately from studies of hospitalized patients. We only included systematic review papers and excluded all commentaries. We abstracted data from the annual reports of notifiable diseases in countries with advanced surveillance systems.

\section{Analytic methods}

Because of the scarcity of information, input data for typhoid and paratyphoid fever were grouped into the 7 Super Regions as defined by the Global Burden of Disease Project (Super Region 1: Australasia, Southern Latin America, High Income North America, High Income Asia Pacific; Super Region 2: Western Europe, Eastern Europe, Central Europe, Central Asia; Super Region 3: Southern Sub-Saharan Africa, Central Sub-Saharan Africa, West Sub-Saharan Africa, East Sub-Saharan Africa; Super Region 4: Northern Africa/Middle East; Super Region 5: South Asia; Super Region 6: East Asia, South East Asia; Super Region 7: Caribbean, Andean Latin America, Central Latin America, Tropical Latin America, Oceania) [7]. We estimated the incidence using data from all eligible studies conducted within the corresponding Super Region and regional groupings. For any Super Region lacking data on paratyphoid fever, we extrapolated an incidence estimate from the Super Region with the closest typhoid fever incidence estimate.

Typhoid fever incidence rates were grouped with respect to age (ie, children $<5$ years and persons $\geq 5$ years) for regions where age-specific data were available. The median proportion of typhoid fever cases observed among children $<5$ years of age was calculated and this figure was used to derive the estimated proportion of cases among those 5 years of age and older. We then calculated age-specific incidence rates and the annual number of typhoid fever episodes within each age strata using the median proportion of typhoid fever cases among each age group and the estimated number of overall typhoid fever episodes across all ages.

To estimate the number of typhoid fever episodes in each Super Region for 2010, we applied the median incidence for each Super Region to the corresponding population estimates. Uncertainty bounds were calculated using interquartile ranges. The total episodes were summed across Super Regions to provide the crude global typhoid fever burden and estimates of uncertainty. 
An adjusted estimate of global typhoid fever burden was also calculated to account for the low sensitivity of the blood culture to isolate S. typhi or S. paratyphi. Similar to previous estimates by Crump et al., an adjustment factor of 2 was chosen based on a conservative estimate of $50 \%$ sensitivity [1]. This figure was the lowest reported sensitivity among 3 studies evaluating this culture method for typhoid fever diagnosis [8-10].

We estimated case-fatality rates for typhoid and paratyphoid fever from the published literature and the surveillance system data and applied to incidence rate estimates to calculate mortality rates.

\section{RESULTS}

The systematic review yielded 24 studies that examined typhoid fever incidence and employed blood culture as the criteria for diagnosis (Figure 1) [11-34]. Five advanced surveillance systems reporting blood-culture confirmed typhoid fever cases were also identified [35-39]. In addition, after the manuscript was accepted, we became aware of one recently published study that met systematic review inclusion criteria, so the analysis was updated to include this data [40]. In total, typhoid fever incidence data was abstracted from 47 countries across 14 (67\%) of the 21 regions (Table 1). Population-based and prospective vaccine studies contributed data for 13 countries across 8 regions. The remaining incidence data was collected by typhoid fever surveillance systems in the 6 developed regions, each of which includes 1 or more countries with national-level surveillance. The developed regions include: High Income Asia Pacific, High Income North America, Central Europe,

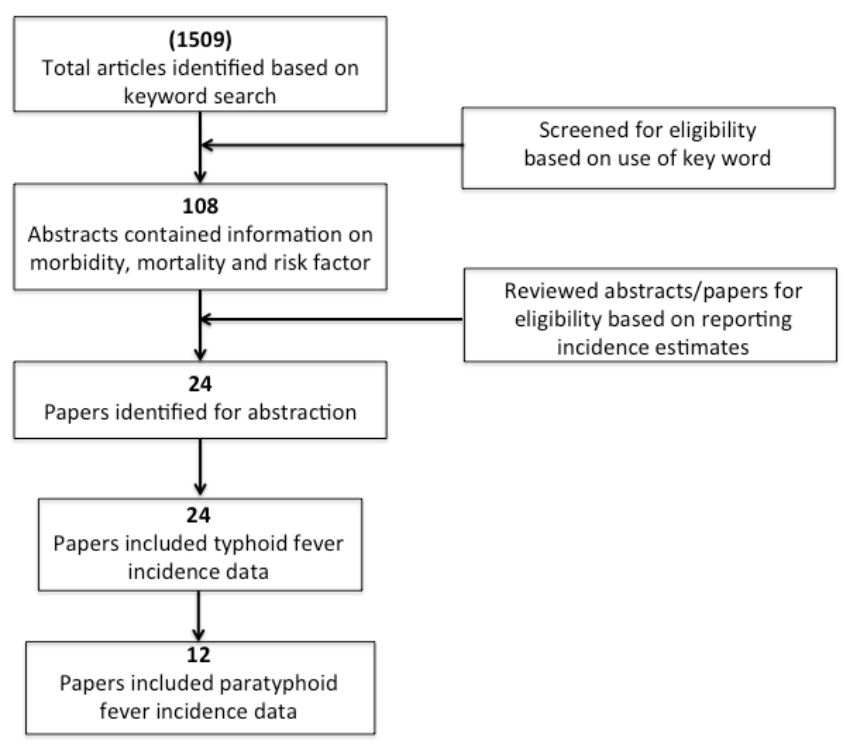

Figure 1 Selection strategy flow diagram used to identify studies on typhoid and paratyphoid fever.
Eastern Europe, Western Europe, and Australia/New Zealand. Overall, our analysis includes national-level incidence data from 34 countries across these regions. Paratyphoid fever incidence data was available for 9 countries representing 7 (33\%) of the 21 regions (Table 2). Only 2 regions included national-level surveillance systems reporting paratyphoid fever incidence (High Income Asia Pacific and Australia/New Zealand). Population-based studies provided paratyphoid fever data for 7 countries in 5 of the regions (Southern Latin America, North Africa/Middle East, South Asia, South East Asia, and East Asia). The median year of data collection for included studies is 2004 .

Input data for typhoid and paratyphoid fever were grouped into 7 Super Regions and median incidence rates and interquartile ranges are presented in Tables 1 and 2, respectively. Paratyphoid fever incidence estimates were extrapolated between Super Regions on the basis of typhoid fever burden estimates. No paratyphoid fever data were available for Super Region 2 (Central Europe, Eastern Europe, Central Asia), Super Region 3 (Southern Sub-Saharan Africa, Central Sub-Saharan Africa, West Sub-Saharan Africa, East Sub-Saharan Africa), and Super Region 7 (Caribbean, Andean Latin America, Central Latin America, Tropical Latin America, Oceania). Extrapolations were made from Super Region 1 (Australia/New Zealand, Southern Latin America, High Income North America, High Income Asia Pacific, Western Europe) to Super Region 2; Super Region 5 (South Asia) to Super Region 3; and Super Region 6 (East Asia and South East Asia) to Super Region 7.

Twenty-two (88\%) of the 25 eligible typhoid fever incidence studies contained age-specific typhoid fever data for children $<5$ years and persons $\geq 5$ and older (Table 3 ). Agespecific data were available for 6 (29\%) of 21 regions representing 5 of 7 Super Regions. All data came from lowand middle-income countries. The median proportion of typhoid fever episodes among children $<5$ years was $57.7 \%$, and among persons $\geq 5$ years, $42.3 \%$. For Super Regions 3 and 5, the median proportion of typhoid fever cases among each age strata was used to calculate regional estimates of annual number of cases and incidence rates for each age group (Table 4).

The median typhoid fever incidence rate for each Super Region applied to the 2010 population estimates generates a crude global estimate of 13474369 typhoid fever episodes each year (Table 4). After adjusting for the low sensitivity of the blood culture typhoid test we estimate typhoid fever incidence to be 26948739 episodes annually.

There is little data to describe typhoid or paratyphoid fever case-fatality rates. In the most recent study on the global typhoid fever burden, Crump et al. assumed a case-fatality rate of $1 \%$ for typhoid fever based on hospital-based data, expert opinion, and mortality rates documented by ad- 
Table 1 Input data for typhoid fever incidence rates and summarized median incidence rates by Super Region* SUPER Riegion

Super Region 1

Australia/New Zealand [35,36]

Latin America, Southern [11-13]

North America, High Income [37]

Asia Pacific, High Income [38] TYPHOID FEVER GASES PERSON-YEARS (P-YEARS) INGIDENGE (EPISODES/1000OO P-YEARS)

Europe, Western [39]

\begin{tabular}{rr}
695 & 17753491 \\
\hline 463 & 7731880 \\
\hline 164 & 136525 \\
\hline 68 & 65718 \\
\hline 28 & 30906 \\
\hline 383 & 1021033000 \\
\hline 31 & 33211134 \\
\hline 207 & 31918266 \\
\hline 50 & 3131242 \\
\hline 35 & 21861785 \\
\hline 832 & 21159333 \\
\hline 468 & 328915133 \\
\hline 38 & 44771106 \\
\hline 51 & 17372910 \\
\hline 219 & 237547356 \\
\hline 1 & 1922572 \\
\hline 1 & 1636715 \\
\hline 192 & 65583388 \\
\hline 140 & 42413512 \\
\hline 98 & 179344312 \\
\hline 108 & 36600283 \\
\hline 1666 & 243565650 \\
\hline 4 & 1242390 \\
\hline 0 & 70524 \\
\hline 36 & 18857776 \\
\hline
\end{tabular}

Median typhoid fever incidence rate per 100000 p-years (IQR) Super Region 2

Europe, Central [39]

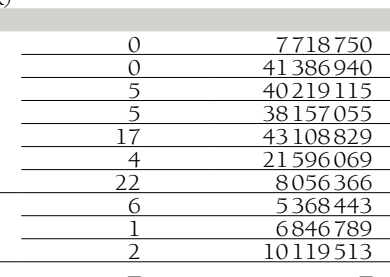

Europe, Eastern [39]

Asia, Central
Median typhoid fever incidence rate per 100000 p-years (IQR)
Super Region 3

Sub-Saharan Africa, Southern [14]

Sub-Saharan Africa, Central

Sub-Saharan Africa, West

Sub-Saharan Africa, East [40]

Median typhoid fever incidence rate per 100000 p-years (IQR)

Super Region 4

North Africa/Middle East [15-17]

Median typhoid fever incidence rate per 100000 p-years (IQR)

Super Region 5

Asia, South [18-27]

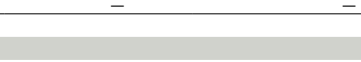

\begin{tabular}{l}
0.1 \\
0.0 \\
\hline
\end{tabular}

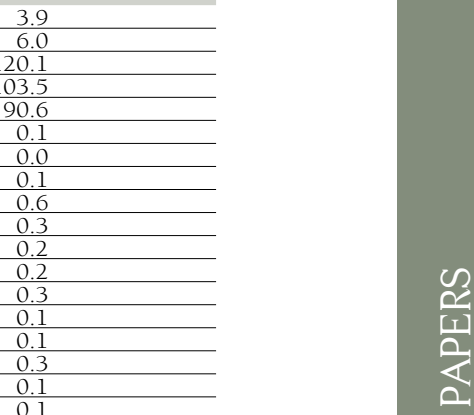

Median typhoid fever incidence rate per 100000 p-years (IQR) Super Region 6

Asia, East $[20,22,23,28]$

Asia, South East [20,22,23,29-32]

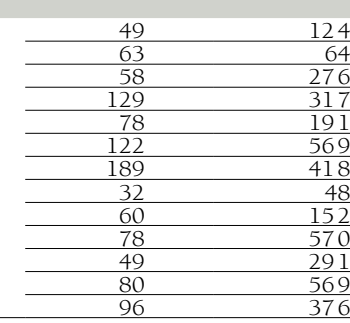

0.1

0.3
0.3
0.1

0.3

0.7
0.0

0.0

$0.3(0.1,0.4)$

0.0

\begin{tabular}{l}
0.0 \\
0.0 \\
0.0 \\
0.0 \\
0.0 \\
0.0 \\
0.3 \\
0.1 \\
0.0 \\
0.0 \\
\hline
\end{tabular}

$<0.1(0,<0.1)$

845.6

$\begin{array}{rr}- & - \\ - & - \\ 794 & 131550\end{array}$

$-\frac{-}{603.6}$ $\begin{array}{lll}<5 \mathrm{y} & \geq 5 \mathrm{y} & \text { All ages } \\ 2552.3^{\dagger} & 366.6^{\dagger} & 724.6(603\end{array}$

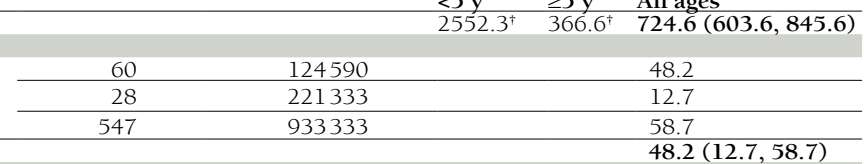

$48.2(12.7,58.7)$

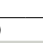

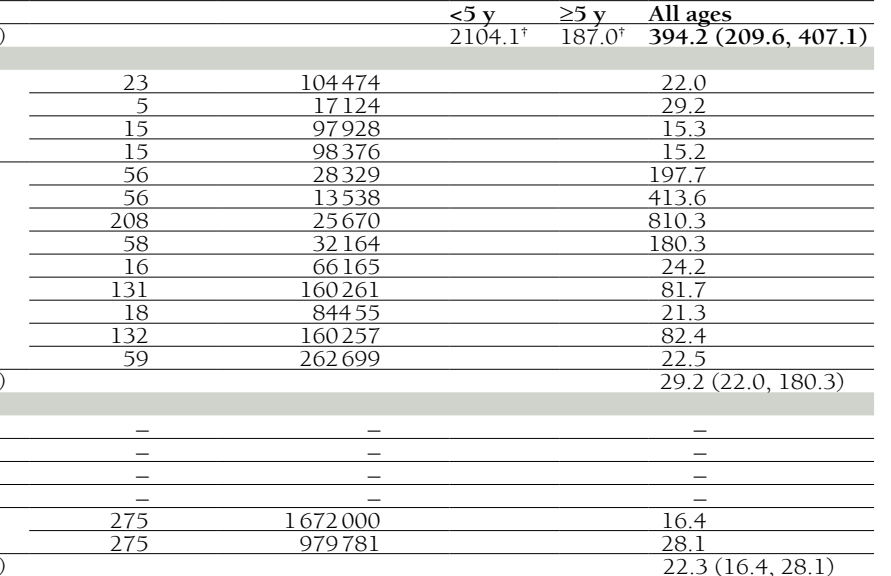

Median typhoid fever incidence rate per 100000 p-years (IQR)

Super Region 7

Laribbean

Latin America, Central

Latin America, Tropical

Oceania $[33,34]$

Median typhoid fever incidence rate per 100000 p-years (IQR)

IQR - interquartile range

* Super Regions as defined by the Global Burden of Disease Project (Super Region 1: Australasia, Southern Latin America, High Income North America, High Income Asia Pacific; Super Region 2: Western Europe, Eastern Europe, Central Europe, Central Asia; Super Region 3: Southern Sub-Saharan Africa, Central Sub-Saharan Africa, West Sub-Saharan Africa, East Sub-Saharan Africa; Super Region 4: Northern Africa/Middle East; Super Region 5: South Asia; Super Region 6: East Asia, South East Asia; Super Region 7: Caribbean, Andean Latin America, Central Latin America, Tropical Latin America, Oceania) [7].

${ }^{\dagger}$ Derived from the following data: estimated annual number of typhoid fever episodes, median proportion of cases $<5$ and $\geq 5$ y of age and age-specific population estimates. 
Table 2 Input data for paratyphoid fever incidence rates and summarized median incidence and mortality rates by Super Region*

\begin{tabular}{|c|c|c|c|c|c|}
\hline \multirow{2}{*}{ Super Region } & \multirow{2}{*}{$\begin{array}{l}\text { ParatyPHOIS FEVIER } \\
\text { CASES }\end{array}$} & \multirow{2}{*}{ Perison-years } & \multirow{2}{*}{$\begin{array}{l}\text { INCIDENCE (EPISODES/ } \\
100000 \text { P-YEARS) }\end{array}$} & $\begin{array}{c}\begin{array}{c}\text { SUPER REGION INCIDENCE } \\
\text { (EPISODES/100 } 000 \text { P-YEARS) }\end{array} \\
\text { MEAUAN (INR) }\end{array}$ & $\begin{array}{l}\text { SUPER REGON MORTALITY } \\
\text { (DEATHS/100 } 000 \text { P-YEARS) }\end{array}$ \\
\hline & & & & MediAn (IOR) & Median (IQR) \\
\hline Super Region 1 & & & & $8.0(0.3,20.6)$ & $<0.1(0,0.1)$ \\
\hline Australia/New Zealand [36] & 471 & 77318800 & 0.6 & & \\
\hline Latin America, Southern $[11,12]$ & 21 & $\begin{array}{r}136525 \\
65718\end{array}$ & $\frac{15.4}{250}$ & & \\
\hline North America, High Income & 11 & $\begin{array}{r}0318 \\
-\end{array}$ & $\frac{25.9}{-}$ & & \\
\hline Asia Pacific, High Income [38] & 219 & 1021033000 & 0.0 & & \\
\hline Europe, Western & - & - & - & & \\
\hline Super Region $2^{\dagger}$ & & & & $8.0(0.3,20.6)$ & $<0.1(0,0.1)$ \\
\hline Europe, Central & - & - & - & & \\
\hline Europe, Eastern & - & - & - & & \\
\hline Asia, Central & - & - & - & & \\
\hline Super Region $3^{+}$ & 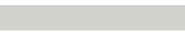 & & & $77.4(42.0,130.3)$ & $0.4(0.2,0.7)$ \\
\hline Sub-Saharan Africa, Southern & - & - & - & & \\
\hline Sub-Saharan Africa, Central & - & - & - & & \\
\hline Sub-Saharan Africa, West & - & - & - & & \\
\hline Sub-Saharan Africa, East & - & - & - & & \\
\hline Super Region 4 & & & & $0.8(\mathrm{~N} / \mathrm{A})$ & $<0.1(\mathrm{~N} / \mathrm{A})$ \\
\hline North Africa/Middle East [17] & 7 & 933333 & 0.8 & & \\
\hline Super Region 5 & & & & $77.4(42.0,130.3)$ & $0.4(0.2,0.7)$ \\
\hline & $\frac{38}{11}$ & $\frac{19161}{15219}$ & $\frac{198.3}{72.3}$ & & \\
\hline & $\frac{11}{24}$ & $\frac{13219}{57075}$ & $\frac{12.3}{42.0}$ & & \\
\hline Asia, South [21,23,25-27] & 7 & 29170 & 24.0 & & \\
\hline & 47 & 56946 & 82.5 & & \\
\hline & 49 & 37608 & 130.3 & & \\
\hline Super Region 6 & & & & $17.9(8.8,27.4)$ & $0.1(0,0.1)$ \\
\hline Asia, East $[23,28]$ & 5 & 104475 & 4.8 & & \\
\hline & 27 & 98376 & 27.4 & & \\
\hline & 3 & 1353830 & 0.2 & & \\
\hline Asia, South East [23,29,31,32] & 48 & $\begin{array}{r}25670 \\
160257\end{array}$ & $\frac{187.0}{12.7}$ & & \\
\hline & $\frac{22}{23}$ & $\frac{160257}{262699}$ & $\frac{13.7}{8.8}$ & & \\
\hline
\end{tabular}

Super Region 7

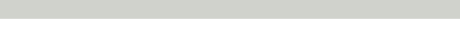

$17.9(8.8,27.4) \quad 0.1(0,0.1)$

Laribbean

Latin America, Central

Latin America, Tropical

Oceania

p-years - person-years, IQR - interquartile range, N/A - not applicable

*Super Regions as defined by the Global Burden of Disease Project (Super Region 1: Australasia, Southern Latin America, High Income North America, High Income Asia Pacific; Super Region 2: Western Europe, Eastern Europe, Central Europe, Central Asia; Super Region 3: Southern Sub-Saharan Africa, Central Sub-Saharan Africa, West Sub-Saharan Africa, East Sub-Saharan Africa; Super Region 4: Northern Africa/Middle East; Super Region 5: South Asia; Super Region 6: East Asia, South East Asia; Super Region 7: Caribbean, Andean Latin America, Central Latin America, Tropical Latin America, Oceania) [7].

${ }^{\dagger}$ Extrapolation used to derive Super Region incidence estimate.

Table 3 An overview of studies with age-specific typhoid fever incidence rates by Super Regions

\begin{tabular}{|c|c|c|c|c|c|c|c|c|}
\hline \multirow[b]{2}{*}{ Super Region* } & \multicolumn{4}{|l|}{$<5 y$} & \multicolumn{4}{|l|}{$\geq 5 y$} \\
\hline & $\begin{array}{l}\text { TYPHOID FEVER } \\
\text { CASES }\end{array}$ & $\begin{array}{l}\text { PensON } \\
\text {-YEARS }\end{array}$ & $\begin{array}{l}\text { INCIDENCE } \\
\text { (CASES/100 } 000 \\
\text { P-YEARS) }\end{array}$ & $\begin{array}{l}\text { PropoRtion } \\
\text { OF OVERALL ( } \%)\end{array}$ & $\begin{array}{l}\text { TYPHOID FEVER } \\
\text { CASES }\end{array}$ & Person-Years & $\begin{array}{l}\text { INCIDENGE } \\
\text { (CASES/100 OOO } \\
\text { P-YEARS) }\end{array}$ & $\begin{array}{l}\text { Proportion of } \\
\text { OVERALL (\%) }\end{array}$ \\
\hline \multirow{2}{*}{ Latin America, Southern [11-13] } & - & - & - & - & 68 & 65718 & 103.5 & N/A \\
\hline & - & - & - & - & 28 & 30906 & 91.0 & N/A \\
\hline Sub-Saharan Africa, East [40] & 240 & 23167 & 1039.9 & 30.3 & 553 & 108383 & 510.3 & 69.7 \\
\hline North Africa / Middle East [15-17] & 9 & 157631 & 5.8 & 7.6 & 545 & 775114 & 70.4 & 92.4 \\
\hline \multirow{6}{*}{ Asia, South [18-27] } & 26 & 1393 & 1870.0 & 89.9 & 23 & 11014 & 210.0 & 10.1 \\
\hline & 28 & 1027 & 2726.4 & 80.9 & 35 & 5427 & 644.9 & 19.1 \\
\hline & 11 & 4061 & 270.9 & 56.4 & 111 & 52885 & 209.8 & 43.6 \\
\hline & 58 & 10118 & 573.2 & 58.1 & 131 & 31727 & 412.9 & 41.9 \\
\hline & 13 & 15545 & 83.6 & 24.0 & 36 & 13625 & 264.2 & 76.0 \\
\hline & 27 & 2089 & 1292.0 & 86.6 & 69 & 34543 & 199.7 & 13.4 \\
\hline \multirow{2}{*}{ Asia, East $[20,22,23,28]$} & 0 & 489 & 0.0 & 0.0 & 23 & 103985 & 22.1 & 100.0 \\
\hline & - & & - & - & 15 & 97928 & 15.3 & N/A \\
\hline \multirow{3}{*}{ Asia, South East $[20,22,23,29-32]$} & 26 & 1989 & 1307.0 & 62.9 & 182 & 23658 & 769.3 & 37.1 \\
\hline & 14 & 12924 & 108.3 & 57.7 & 117 & 147337 & 79.4 & 42.3 \\
\hline & - & - & - & - & 18 & 84455 & 21.3 & N/A \\
\hline $\begin{array}{l}\text { Median proportion of cases split by } \\
<5 y \text { and } 5 \text { y of age and older for low } \\
\text { and middle income countries }\end{array}$ & & & & 57.7 & & & & $42.3^{+}$ \\
\hline
\end{tabular}

p-years - person-years, IQR - interquartile range, N/A - not applicable, y - years

* Super Regions as defined by the Global Burden of Disease Project (Super Region 1: Australasia, Southern Latin America, High Income North America, High Income Asia Pacific; Super Region 2: Western Europe, Eastern Europe, Central Europe, Central Asia; Super Region 3: Southern Sub-Saharan Africa, Central Sub-Saharan Africa, West Sub-Saharan Africa, East Sub-Saharan Africa; Super Region 4: Northern Africa/Middle East; Super Region 5: South Asia; Super Region 6: East Asia, South East Asia; Super Region 7: Caribbean, Andean Latin America, Central Latin America, Tropical Latin America, Oceania) [7].

'Derived from the median proportion of overall cases attributable to children under $5 \mathrm{y}$ of age. 
Table 4 Annual number of typhoid fever episodes, 2010 by Super Region*

\begin{tabular}{|c|c|c|c|}
\hline Super Region & 2010 & & \\
\hline Super Region 1 & All ages & & \\
\hline Population [41] & 1019736630 & & \\
\hline Median typhoid fever incidence rate per 100000 p-years (IQR) & $0.3(0.1,0.4)$ & & \\
\hline Annual number of typhoid fever episodes (IQR) & $3059(1019,4078)$. & & \\
\hline Super Region 2 & All ages & & \\
\hline Population [41] & 406303917 & & \\
\hline Median typhoid fever incidence rate per 100000 p-years (IQR) & $<0.1(0,<0.1)$ & & \\
\hline Annual number of typhoid fever episodes (IQR) & $406(0,406)$ & & \\
\hline Super Region 3 & $<5 \mathrm{y}$ & $\geq 5$ years & All ages \\
\hline Population [41] & 140250136 & 715910880 & 856161016 \\
\hline Median typhoid fever incidence rate per 100000 p-years (IQR) & 2552.3 & 366.6 & $724.6(603.6,845.6)$ \\
\hline Annual number of typhoid fever episodes (IQR) & $3579559^{+}$ & $2624183^{+}$ & $6203742(5167787,7239697)$ \\
\hline Super Region 4 & All ages & & \\
\hline Population [41] & 445487756 & & \\
\hline Median typhoid fever incidence rate / 100000 p-years (IQR) & $48.2(12.7,58.7)$ & & \\
\hline Annual number of typhoid fever episodes (IQR) & $214725(56576,261501)$ & & \\
\hline Super Region 5 & $<5 y$ & $\geq 5 \mathrm{y}$ & All ages \\
\hline Population [41] & 174016500 & 1435769400 & 1609785900 \\
\hline Median typhoid fever incidence rate per 100000 p-years (IQR) & 2104.1 & 187.0 & $394.2(209.6,407.1)$ \\
\hline Annual number of typhoid fever episodes (IQR) & $3661512^{\dagger}$ & $2684263^{\dagger}$ & $6345776(3374111,6553438)$ \\
\hline Super Region 6 & All ages & & \\
\hline Population [41] & 2016815598 & & \\
\hline Median typhoid fever incidence rate per 100000 p-years (IQR) & $29.2(22.0,180.3)$ & & \\
\hline Annual number of typhoid fever episodes (IQR) & $588910(443699,3636318)$ & & \\
\hline Super Region 7 & All ages & & \\
\hline Population [41] & 528026317 & & \\
\hline Median typhoid fever incidence rate per 100000 p-years (IQR) & $22.3(16.4,28.1)$ & & \\
\hline Annual number of typhoid fever episodes (IQR) & $117749(86596,148375)$ & & \\
\hline $\begin{array}{l}\text { Global Total: annual number of typhoid fever episodes } \\
\text { (crude (IQR) / adjusted (IQR) } \ddagger \text { ) }\end{array}$ & 13474369 (9129791, 17843 & 16) / 26948738 & $18259583,35687632)$ \\
\hline
\end{tabular}

\section{p-years - person-years, IQR - interquartile range}

* Super Regions as defined by the Global Burden of Disease Project (Super Region 1: Australasia, Southern Latin America, High Income North America, High Income Asia Pacific; Super Region 2: Western Europe, Eastern Europe, Central Europe, Central Asia; Super Region 3: Southern Sub-Saharan Africa, Central SubSaharan Africa, West Sub-Saharan Africa, East Sub-Saharan Africa; Super Region 4: Northern Africa/Middle East; Super Region 5: South Asia; Super Region 6: East Asia, South East Asia; Super Region 7: Caribbean, Andean Latin America, Central Latin America, Tropical Latin America, Oceania) [7]

'Derived from median proportion of cases $<5$ and $\geq 5$ y of age (Table 5 ).

¥Adjusted to account for low sensitivity of blood culture typhoid test.

vanced national surveillance systems [1]. Given we found no new data to suggest an improvement in typhoid fever case fatality rates, we also used this figure to estimate the total number of annual deaths and to derive mortality estimates, which are presented in Table 5. Past studies on the global paratyphoid fever burden have not reported mortality estimates. Our study assumed a case-fatality rate of $0.5 \%$ given that paratyphoid fever is generally less severe than typhoid fever [42]. Mortality estimates for paratyphoid fever are presented in Table 2.

\section{DISCUSSION}

Our results suggest that in 2010, there were an estimated 13.5 million typhoid fever episodes globally. This estimate is comparable to the 2000 crude estimate of 10.8 million episodes published by Crump et al [1]. We sought to update the previous estimate and in doing so, found a number of more recently published studies with higher incidence rates than those reported in older studies that influenced our final estimate. We used slightly different inclusion and exclusion criteria and applied slightly different methods for estimating incidence globally from the previous systematic review, which all contributed to the observed differences. However, given that the world's population has grown by $10 \%$ in the last 10 years, our revised estimate, compared to the previously published 2000 estimate, is well within a plausible margin of error.
Quantity of source data remains a major limitation for estimating the global burden of typhoid and paratyphoid fever. While additional data on paratyphoid fever is needed across all regions, typhoid fever estimates are limited by the scarcity of reliable incidence data from many of the developing regions in particular. Lacking surveillance systems or eligible population-based studies, typhoid fever incidence data were unavailable for 7 (33\%) regions including: Central Asia, Central Sub-Saharan Africa, West Sub-Saharan Africa, Caribbean, Andean Latin America, Central Latin America, and Tropical Latin America. Furthermore, incidence estimates for several regions were based on few studies. Of note, we identified only 5 eligible studies conducted in Africa. As a result, our estimate for Super Region 3 - representing all of sub-Saharan Africa - was based on only two studies conducted in South Africa and Kenya $[14,40]$. Similarly, North Africa/Middle East estimates relied on only 3 studies that were carried out in Egypt [1517]. Outside of Africa, there is also limited data available for Super Region 7. Only 2 studies from Fiji and Tonga were used to estimate the burden of disease for this region and both reported the results of pilot surveillance systems, thus there exists considerable uncertainty related to this approximation [33,34]. Additional population-based surveillance studies must be carried out in Africa and other developing regions to develop a more accurate understanding of the global typhoid fever burden. 
Table 5 Summarized median typhoid fever mortality rates by Super Region*

\begin{tabular}{|c|c|c|}
\hline \multirow[t]{2}{*}{ Super Region } & \multirow[t]{2}{*}{ Region } & 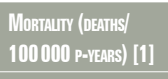 \\
\hline & & Median (IOR) \\
\hline \multirow{5}{*}{ Super Region 1} & Australia/New Zealand $[35,36]$ & \multirow{5}{*}{$<0.1(0,<0.1)$} \\
\hline & Latin America, Southern [11-13] & \\
\hline & North America, High Income [37] & \\
\hline & Asia Pacific, High Income [38] & \\
\hline & Europe, Western [39] & \\
\hline \multirow{3}{*}{ Super Region 2} & Europe, Central [39] & \multirow{3}{*}{$<0.1(0,<0.1)$} \\
\hline & Europe, Eastern [39] & \\
\hline & Asia, Central & \\
\hline \multirow{4}{*}{ Super Region 3} & Sub-Saharan Africa, Southern [14] & \multirow{4}{*}{$7.2(6.0,8.5)$} \\
\hline & Sub-Saharan Africa, Central & \\
\hline & Sub-Saharan Africa, West & \\
\hline & Sub-Saharan Africa, East [40] & \\
\hline Super Region 4 & North Africa/Middle East [15-17] & $0.5(0.1,0.6)$ \\
\hline Super Region 5 & Asia, South [18-27] & $3.9(2.1,4.1)$ \\
\hline Super Region 6 & $\begin{array}{l}\text { Asia, East }[20,22,23,28] \\
\text { Asia, South East }[20,22,23,29-32]\end{array}$ & 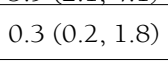 \\
\hline \multirow{5}{*}{ Super Region 7} & Caribbean & \multirow{5}{*}{$0.2(0.2,0.3)$} \\
\hline & Latin America, Andean & \\
\hline & Latin America, Central & \\
\hline & Latin America, Tropical & \\
\hline & Oceania $[33,34]$ & \\
\hline
\end{tabular}

p-years - person-years, IQR - interquartile range

* Super Regions as defined by the Global Burden of Disease Project (Super Region 1: Australasia, Southern Latin America, High Income North America, High Income Asia Pacific; Super Region 2: Western Europe, Eastern Europe, Central Europe, Central Asia; Super Region 3: Southern Sub-Saharan Africa, Central Sub-Saharan Africa, West Sub-Saharan Africa, East SubSaharan Africa; Super Region 4: Northern Africa/Middle East; Super Region 5: South Asia; Super Region 6: East Asia, South East Asia; Super Region 7: Caribbean, Andean Latin America, Central Latin America, Tropical Latin America, Oceania) [7]

We restricted our analysis to studies and surveillance systems that used blood culture as the criteria for diagnosis. Although typhoid and paratyphoid fever are most commonly diagnosed using this method, it is only 50\% sensitive. Factors that influence test sensitivity include antimicrobial use, the volume of blood collected, and the timing of blood collection $[8,10,43]$. These important limitations introduce a bias toward underestimation. In contrast, the inclusion of vaccine studies as a source for incidence data promotes a bias toward overestimation as sites are generally selected for having high incidence rates due to sample size considerations.

Typhoid and paratyphoid fever are major public health problems, especially in the developing world. Our study reports a revised estimate of the global burden of these diseases based on new data available from recent populationbased studies and broader coverage of surveillance systems. In total, we identified 49 sources of new data that have become available since the 2000 estimate published by Crump et al. in 2004 [1]: 15 population-based studies, 30 national-surveillance systems, and 4 partially representative surveillance systems. Collectively, these sources provide estimates of overall typhoid fever incidence rates from 14 (67\%) of the 21 regions across 5 Super Regions.

Although our understanding of the global burden of these diseases has improved with more recent data, both enteric fevers remain poorly quantified. Critical gaps in our understanding persist, as the burden remains largely unknown in many of the regions. Appreciable gains would be made by: a) developing improved diagnostic methods; b) implementing surveillance systems; and c) carrying out additional population-based research, particularly in subSaharan Africa and other developing countries. Recent studies have shown that paratyphoid fever accounts for an increasing proportion of enteric fever in several regions $[19,23,44-47]$. If this trend continues, important challenges can be anticipated in the absence of an effective vaccine for this disease. In addition, multi-drug resistant S. typhi and S. paratyphi organisms may continue to increase in prevalence and could certainly hamper efforts to reduce related morbidities. An accurate epidemiological profile of the global burden of typhoid and paratyphoid fever is important to developing effective disease prevention and control strategies.

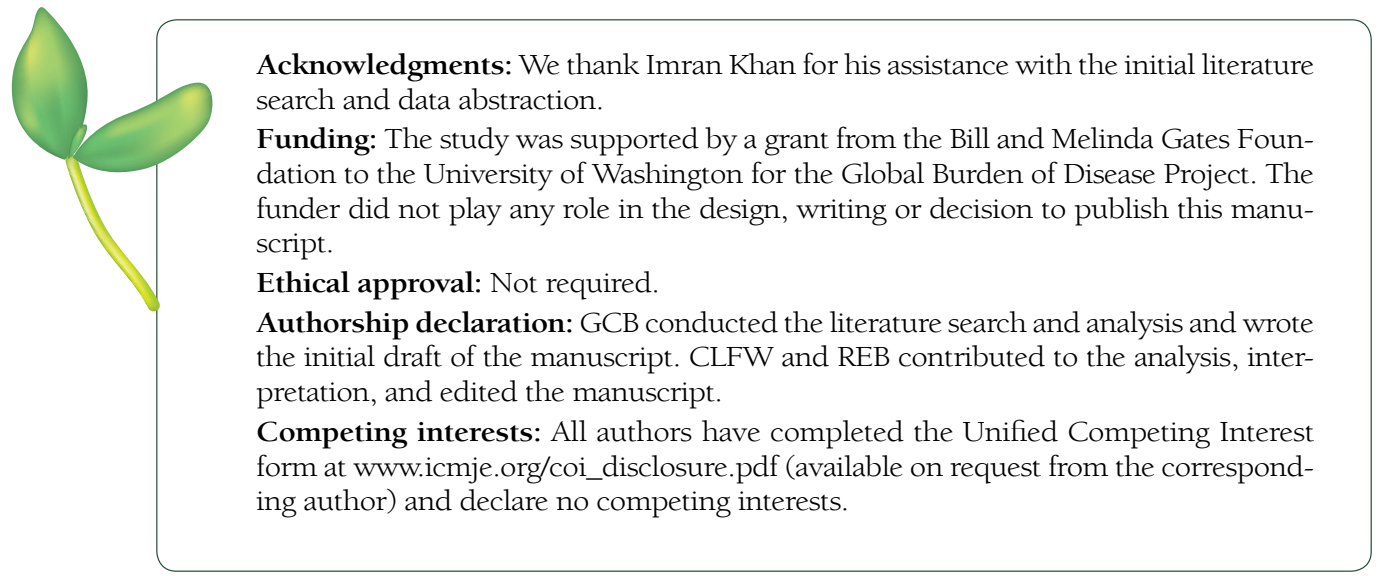


1 Crump JA, Luby SP, Mintz ED. The global burden of typhoid fever. Bull World Health Organ. 2004;82:34653. Medline: 15298225

2 Stuart BM, Pullen RL. Typhoid; clinical analysis of 360 cases. Arch Intern Med (Chic). 1946;78:629-61. Medline:20278487

3 Edelman R, Levine MM. Summary of an international workshop on typhoid fever. Rev Infect Dis. 1986;8:32949. Medline:3726393 doi:10.1093/clinids/8.3.329

4 World Health Organization. The world health report: Fighting disease, fostering development. Geneva: World Health Organization; 1996. p 137.

5 Committee on Issues and Priorities for New Vaccine Development, Division of Health Promotion and Disease Prevention, Division of International Health. New vaccine development: Establishing priorities: Diseases of importance in developing countries. Washington (DC): National Academy Press; 1986. p 432.

6 Kothari A, Pruthi A, Chugh TD. The burden of enteric fever. J Infect Dev Ctries. 2008;2:253-9. Medline:19741285

7 Global Burden of Disease Study [Internet]. Seattle (WA): Institute for Health Metrics and Evaluation; c2012. Available at: http://www.globalburden.org/. Accessed: 3 March 2012.

8 Hoffman SL, Edman DC, Punjabi NH, Lesmana M, Cholid A, Sundah S, et al. Bone marrow aspirate culture superior to streptokinase clot culture and $8 \mathrm{ml} \mathrm{1:10} \mathrm{blood-to-broth} \mathrm{ratio} \mathrm{blood} \mathrm{culture} \mathrm{for} \mathrm{diagnosis} \mathrm{of} \mathrm{typhoid}$ fever. Am J Trop Med Hyg. 1986;35:836-9. Medline:3089041

9 Gilman RH, Terminel M, Levine MM, Hernandez-Mendoza P, Hornick RB. Relative efficacy of blood, urine, rectal swab, bone-marrow, and rose-spot cultures for recovery of Salmonella typhi in typhoid fever. Lancet. 1975;1:1211-3. Medline:48834 doi:10.1016/S0140-6736(75)92194-7

10 Wain J, Pham VB, Ha V, Nguyen NM, To SD, Walsh AL, et al. Quantitation of bacteria in bone marrow from patients with typhoid fever: relationship between counts and clinical features. J Clin Microbiol. 2001;39:15716. Medline:11283089 doi:10.1128/JCM.39.4.1571-1576.2001

11 Black RE, Levine MM, Ferreccio C, Clements ML, Lanata C, Rooney J, et al. Efficacy of one or two doses of Ty2 la Salmonella typhi vaccine in enteric-coated capsules in a controlled field trial. Chilean Typhoid Committee. Vaccine. 1990;8:81-4. Medline:2180234 doi:10.1016/0264-410X(90)90183-M

12 Levine MM, Ferreccio C, Black RE, Germanier R. Large-scale field trial of Ty2la live oral typhoid vaccine in enteric-coated capsule formulation. Lancet. 1987;1:1049-52. Medline:2883393 doi:10.1016/S01406736(87)90480-6

13 Levine MM, Ferreccio C, Cryz S, Ortiz E. Comparison of enteric-coated capsules and liquid formulation of Ty2 la typhoid vaccine in randomised controlled field trial. Lancet. 1990;336:891-4. Medline:1976928 doi:10.1016/0140-6736(90)92266-K

14 Klugman KP, Gilbertson IT, Koornhof HJ, Robbins JB, Schneerson R, Schulz D, et al. Protective activity of Vi capsular polysaccharide vaccine against typhoid fever. Lancet. 1987;2:1165-9. Medline:2890805 doi:10.1016/ S0140-6736(87)91316-X

15 Wahdan MH, Serie C, Cerisier Y, Sallam S, Germanier R. A controlled field trial of live Salmonella typhi strain Ty 21 a oral vaccine against typhoid: three-year results. J Infect Dis. 1982;145:292-5. Medline:7037982 doi:10.1093/infdis/145.3.292

16 Crump JA, Youssef FG, Luby SP, Wasfy MO, Rangel JM, Taalat M, et al. Estimating the incidence of typhoid fever and other febrile illnesses in developing countries. Emerg Infect Dis. 2003;9:539-44. Medline:12737736

17 Srikantiah P, Girgis FY, Luby SP, Jennings G, Wasfy MO, Crump JA, et al. Population-based surveillance of typhoid fever in Egypt. Am J Trop Med Hyg. 2006;74:114-9. Medline:16407354

18 Brooks WA, Hossain A, Goswami D, Nahar K, Alam K, Ahmed N, et al. Bacteremic typhoid fever in children in an urban slum, Bangladesh. Emerg Infect Dis. 2005;11:326-9. Medline:15752457 doi:10.3201/ eid1102.040422

19 Sinha A, Sazawal S, Kumar R, Sood S, Reddaiah VP, Singh B, et al. Typhoid fever in children aged less than 5 years. Lancet. 1999;354:734-7. Medline:10475185 doi:10.1016/S0140-6736(98)09001-1

20 Chen X, Stanton B, Pach A, Nyamete A, Ochiai RL, Kaljee L, et al. Adults' perceived prevalence of enteric fever predicts laboratory-validated incidence of typhoid fever in children. J Health Popul Nutr. 2007;25:469-78. Medline:18402191

21 Khan MI, Sahito SM, Khan MJ, Wassan SM, Shaikh AW, Maheshwari AK, et al. Enhanced disease surveillance through private health care sector cooperation in Karachi, Pakistan: experience from a vaccine trial. Bull World Health Organ. 2006;84:72-7. Medline:16501718 doi:10.2471/BLT.05.023630

22 Ochiai RL, Acosta CJ, Danovaro-Holliday MC, Baiqing D, Bhattacharya SK, Agtini MD, et al. A study of typhoid fever in five Asian countries: disease burden and implications for controls. Bull World Health Organ. 2008;86:260-8. Medline:18438514 doi:10.2471/BLT.06.039818

23 Ochiai RL, Wang X, von Seidlein L, Yang J, Bhutta ZA, Bhattacharya SK, et al. Salmonella paratyphi A rates, Asia. Emerg Infect Dis. 2005;11:1764-6. Medline:16318734 doi:10.3201/eid1111.050168

24 Acharya IL, Lowe CU, Thapa R, Gurubacharya VL, Shrestha MB, Cadoz M, et al. Prevention of typhoid fever in Nepal with the Vi capsular polysaccharide of Salmonella typhi. A preliminary report. N Engl J Med. 1987;317:1101-4. Medline:3657877 doi:10.1056/NEJM198710293171801

25 Sur D, Ali M, von Seidlein L, Manna B, Deen JL, Acosta CJ, et al. Comparisons of predictors for typhoid and paratyphoid fever in Kolkata, India. BMC Public Health. 2007;7:289. Medline:17935611 doi:10.1186/14712458-7-289 
26 Sur D, Ochiai RL, Bhattacharya SK, Ganguly NK, Ali M, Manna B, et al. A cluster-randomized effectiveness trial of Vi typhoid vaccine in India. N Engl J Med. 2009;361:335-44. Medline:19625715 doi:10.1056/NEJMoa0807521

27 Siddiqui FJ, Rabbani F, Hasan R, Nizami SQ, Bhutta ZA. Typhoid fever in children: some epidemiological considerations from Karachi, Pakistan. Int J Infect Dis. 2006;10:215-22. Medline:16431148 doi:10.1016/j. ijid.2005.03.010

28 Yang HH, Wu CG, Xie GZ, Gu QW, Wang BR, Wang LY, et al. Efficacy trial of Vi polysaccharide vaccine against typhoid fever in south-western China. Bull World Health Organ. 2001;79:625-31. Medline:11477965

29 Lin FY, Ho VA, Khiem HB, Trach DD, Bay PV, Thanh TC, et al. The efficacy of a Salmonella typhi Vi conjugate vaccine in two-to-five-year-old children. N Engl J Med. 2001;344:1263-9. Medline:11320385 doi:10.1056/ NEJM200104263441701

30 Lin FY, Vo AH, Phan VB, Nguyen TT, Bryla D, Tran CT, et al. The epidemiology of typhoid fever in the Dong Thap Province, Mekong Delta region of Vietnam. Am J Trop Med Hyg. 2000;62:644-8. Medline:11289678

31 Simanjuntak CH, Paleologo FP, Punjabi NH, Darmowigoto R, Soeprawoto, Totosudirjo H, et al. Oral immunisation against typhoid fever in Indonesia with Ty2la vaccine. Lancet. 1991;338:1055-9. Medline:1681365 doi:10.1016/0140-6736(91)91910-M

32 Vollaard AM, Ali S, Widjaja S, Asten HA, Visser LG, Surjadi C, et al. Identification of typhoid fever and paratyphoid fever cases at presentation in outpatient clinics in Jakarta, Indonesia. Trans R Soc Trop Med Hyg. 2005;99:440-50. Medline:15837356 doi:10.1016/j.trstmh.2004.09.012

33 Dunn J, Pryor J, Saketa S, Wasale D, Buadromo E, Kishore K, et al. Laboratory-based Salmonella surveillance in Fiji, 2004-2005. Pac Health Dialog. 2005;12:53-9. Medline:18181494

34 Lutui T. 'Ofanoa M, Finau S, Maika KV. Typhoid fever in Tonga. Pac Health Dialog. 1999;6:240-4.

35 The OzFoodNet Working Group. Monitoring the incidence and causes of diseases potentially transmitted by food in Australia: Annual report of the OzFoodNet Network, 2009. Commun Dis Intell. 2010;34:396-426. Medline:21413526

36 Institute of Environmental Science and Research Limited, Population and Environmental Health Group. Notifiable and other diseases in New Zealand: 2009 annual surveillance report. Porirua (NZ): Institute of Environmental Science and Research Limited; 2010 April. 63 p. ISSN: 11793058.

37 Centers for Disease Control and Prevention. Summary of notifiable diseases - United States, 2008 for Morbidity and Mortality Weekly Report. Atlanta (GA): Centers for Disease Control and Prevention; 2010. p 94.

38 Infectious Disease Surveillance Center. Infectious Agents Surveillance Report: Typhoid fever and paratyphoid fever in Japan [Internet]. 2009 [cited 2011 April 15]; Available at: http://idsc.nih.go.jp/iasr/index.html.

39 European Centre for Disease Prevention and Control (ECDC). The European Surveillance System (TESSy). Stockholm (SE): ECDC; 2011.

40 Breiman RF, Cosmas L, Njuguna H, Audi A, Olack B, Ochieng JB, et al. Population-based incidence of typhoid fever in an urban informal settlement and a rural area in Kenya: Implications for typhoid vaccine use in Africa. PLoS ONE. 2012;7:e29119. Medline:22276105 doi:10.1371/journal.pone.0029119

41 United Nations, Department of Economic and Social Affairs, Population Division, Population Estimates and Projections Section. World population prospects, the 2006 revision. New York: United Nations; 2007. 96 p.

42 Bhan MK, Bahl R, Bhatnagar S. Typhoid and paratyphoid fever. Lancet. 2005;366:749-62. Medline:16125594 doi:10.1016/S0140-6736(05)67181-4

43 Butler T, Knight J, Nath SK, Speelman P, Roy SK, Azad MA. Typhoid fever complicated by intestinal perforation: a persisting fatal disease requiring surgical management. Rev Infect Dis. 1985;7:244-56. Medline:3890097 doi:10.1093/clinids/7.2.244

44 Shlim DR, Schwartz E, Eaton M. Clinical Importance of Salmonella Paratyphi A Infection to Enteric Fever in Nepal. J Travel Med. 1995;2:165-8. Medline:9815378 doi:10.1111/j.1708-8305.1995.tb00645.x

45 Sood S, Kapil A, Dash N, Das BK, Goel V, Seth P. Paratyphoid fever in India: An emerging problem. Emerg Infect Dis. 1999;5:483-4. Medline:10341194 doi:10.3201/eid0503.990329

46 Tankhiwale SS, Agrawal G, Jalgaonkar SV. An unusually high occurrence of Salmonella enterica serotype paratyphi A in patients with enteric fever. Indian J Med Res. 2003;117:10-2. Medline:12866820

47 Woods CW, Murdoch DR, Zimmerman MD, Glover WA, Basnyat B, Wolf L, et al. Emergence of Salmonella enterica serotype Paratyphi A as a major cause of enteric fever in Kathmandu, Nepal. Trans R Soc Trop Med Hyg. 2006;100:1063-7. Medline:16714040 doi:10.1016/j.trstmh.2005.12.011 